Von Annette Piening as letzte und wohl gravierendste Zugeständnis an die Atomindustrie ist der Kompromißvorschlag aus dem Bundeswirtschaftsministerium: freiwillige, entschädigungsfreie Stillegungen auf der einen Seite sollen durch kartellrechtliche Zurickhaltung bei Fusionen und eine viel zu niedrigen Deckelung der Quoten fuir Kraft-Wärme-Kopplung und erneuerbare Energien zum Schutz der alten Monopolunternehmen auf dem liberalisierten Strommarkt auf der anderen Seite erkauft werden.

Spätestens an diesem Punkt stellt sich dem ernsthaften Verfechter einer Energiewende die Frage, ob es das wert sein kann. Denn wie ein tieferer Blick in die Bilanzbïcher der Atomindustrie zeigt, bläst der Atomindustrie der Wind schon seit Jahren von ganz anderer Seite kräftig ins Gesicht. Während es wohl keinen Industriezweig in Deutschland gibt, der sich in der Öfentlichkeit stärker ïber staatliche Interventionen beklagt, ist die kommerzielle Kernenergienutzung schon seit ihrem Beginn durch die Realität technischer und betriebswirtschaftlicher Unzulänglichkeiten vom Niedergang bedroht.

Der Auftragseingang für Atomkraftwerke hat weltweit bereits 1975 - keine fünf Jahre nach Fertigstellung der ersten Leistungsreaktoren seinen Höhepunkt überschritten und 1987 den Stand von vor 1965 erreicht. Auch der vermeintliche Aufschwung durch Nachrïstungsbedarf in Osteuropa konnte den Niedergang nicht verhindern. Und von den lediglich 31 Ländern, die weltweit Atomanlagen betreiben, haben heute nur drei halbwegs ernstzunehmende Ausbaupläne. In der Folge ist der nukleare Geschäftsbereich in der Kraftwerkssparte von Siemens seit Beginn der 90er Jahre von 50 auf 20 Prozent des Umsatzes zusammengeschmolzen.

Aber selbst während der kurzen Blïte der Atomindustrie verzögerte sich die Abwicklung der Aufträge, soweit sie nicht storniert wurden, oft über Jahre. Und anders als von der Atomindustrie gerne behauptet wird, geschah dies durchaus nicht aufgrund eines ,ausstiegsorientierten Genehmigungsvollzuges“ der Behörden. Wie die grindliche Analyse belegt, ist die Entwicklung der Kernenergie vor allem durch wirtschaftliche Fehlschläge geprägt: In Ländern wie den LSA und auch in Deutschland waren es ganz wesentlich betriebswirtschaftliche Fehlkalkulationen und gravierendes Mißmanagement bei Planung und Bau der Kraftwerke, wodurch die Kosten in die Höhe schnellten und
Der gezielte Einstieg in die Energiewende könnte den Atomausstieg beschleunigen

\section{Wo bleibt die Politik?}

\section{Auch mehr als ein halbes Jahr nach} Aufnahme der Konsensgespräche zwischen der rot-grünen Bundesregierung und den Atomkraftwerksbetreibern scheint eine Einigung in Sachen Atomausstieg in weiter Ferne. Um trotz zunehmend verhärteter Fronten einer Einigung nöherzukommen, werden immer größere Zugeständnisse an die Stromindustrie in die Diskussion gebracht. Diese gefährden jedoch die Energiewende. Eine Änderung der politischen Strategie könnte hingegen zwei Fliegen mit einer Klappe schlagen.

der weitere Kernenergie-Ausbau begrenzt wurde. Genehmigungsauflagen und Proteste aus der Bevölkerung waren stets nur ein Faktor unter mehreren und selten der dominierende. Selbst der vermeintliche Erfolg der Kernkraft in Frankreich war nur mit massiven Subventionen möglich, deren Last durch die kommenden Generationen abgetragen werden muß.

Auch der Betrieb der Anlagen ist durchaus keine Erfolgsstory. Obgleich die deutschen Betreiber hente die hohen Verfiggbarkeitswerte hervorheben, so sind bei der Mehrzahl der Anlagen sicherheitsrelevante Nachrüstungen fuir lange Stillstandszeiten und hohe Kosten verantwortlich. Und daß die erste Generation der Leistungsgeneratoren das Ende ihrer Lebensdauer erreicht hat, demonstriert die zunehmende Zahl der endgültigen Außerbetriebnahmen: in den westlichen Industrienationen wurden 1997 und 199812 Reaktoren aus betriebswirtschaftlichen Gründen stillgelegt, genauso viele wie in den sieben Jahren zuvor. Nur zwei der Anlagen liefen länger als 25 und keine über 31 Jahre.

Damit nicht genug: Wie sich nun herausstellt, hat die Kernenergie auch in liberalisierten Strommärkten auf lange Sicht keine Chancen: Die britische Regierung hat über Jahre ohne Erfolg ihre Atomkraftwerke wie Sauerbier auf dem freien Markt angeboten und finanziert sie nun über eine Stromsteuer auf klimaschädigende Brennstoffe. Auf den liberalisierten Strommärkten in den USA zählen Investitionen in Atommeiler zu den ,stranded costs“ und kommen in den Genuß großzïgiger Ausnahmeregelungen zum Schutz gegen die Gnadenlosigkeit des freien Marktes. Was die zukünftige Versorgung angeht, so sind aus betriebswirtschaftlicher Sicht Gasturbinen und dezentrale Systeme die erste Wahl. Alle Anzeichen weisen darauf hin, daß es sich bei der Großtechnik Kernenergie um ein Auslaufmodell handelt.

\section{Historische Chance nutzen}

Ohne das wichtige Ziel eines politisch beschleunigten Endes der Atomenergienutzung in Abrede stellen zu wollen, sollte aus diesen Erkenntnissen eine entscheidende Konsequenz für eine energiepolitische Strategie gezogen werden: Die historische Chance, die sich im Zuge der liberalisierung der Energiemärkte für die Neugestaltung der energiepolitischen Rahmenhedingungen im Sinne einer Energiewende ergibt, sollte auf jeden Fall wahrgenommen werden:

Durch eine gesetzliche Regelung und offensive Umsetzung eines diskriminierungsfreien Netzzugangs,

durch die Förderung regenerativer Energien mittels einer marktorientierten Mindestvergiitung oder ansteigender (!) Quoten,

durch einen konsequenten Hemmnisabbau für die Kraft-Wärme-Kopplung, sowie

durch gezielte Unterstiitzung von Energieeffizienz in allen Bereichen.

Wie sich in den derzeit tobenden Preiskämpfen bereits abzeichnet, besteht andernfalls die ernsthafte Gefahr, daß auf einem liberalisierten Strommarkt vor allem die Interessen der großen Stromunternehmen vertreten würden. Cranz im Zeichen der Kontinuität deutscher (Atom)energiepolitik wïrde sich deren Monopolstellung vor der Liberalisierung in eine marktbeherrschende Stellung nach der Liberalisierung verwandeln. Am Ende könnten wir mit leeren Händen dastehen, ohne Konsens und ohne Energiewende.

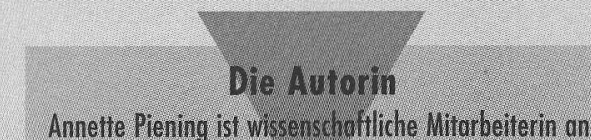
der Forschungsstelle für Umweltpolitik (FFU), Berlin. Kontakt: FFU, Freie Universität Berlin, lhnesir. 22, 14195 Berlin. Tel.030/ 838-4490, Fax-6685, E-mail: piening@zedat.fu-berlin.de 
(c) 20I0 Authors; licensee IÖW and oekom verlag. This is an article distributed under the terms of the Creative Commons Attribution Non-Commercial No Derivates License (http://creativecommons.org/licenses/by-nc-nd/3.o/), which permits unrestricted use, distribution, and reproduction in any medium, provided the original work is properly cited. 